\title{
BMJ Open Low-glycaemic index diet to improve glycaemic control and cardiovascular disease in type 2 diabetes: design and methods for a randomised, controlled, clinical trial
}

Laura Chiavaroli, ${ }^{1,2}$ Arash Mirrahimi, ${ }^{2,3}$ Christopher Ireland, ${ }^{1,2}$ Sandra Mitchell, ${ }^{1,2}$ Sandhya Sahye-Pudaruth, ${ }^{1,2}$ Judy Coveney, ${ }^{2}$ Omodele Olowoyeye, ${ }^{4,5}$ Tishan Maraj, ${ }^{4,5}$ Darshna Patel, ${ }^{1,2}$ Russell J de Souza, ${ }^{2,6}$ Livia S A Augustin,, ${ }^{2,7}$ Balachandran Bashyam, ${ }^{1,2}$ Sonia Blanco Mejia, ${ }^{1,2}$ Stephanie K Nishi, ${ }^{1,2}$ Lawrence A Leiter, ${ }^{1,8}$ Robert G Josse ${ }^{1,8}$ Gail McKeown-Eyssen, ${ }^{9}$ Alan R Moody, ${ }^{4,5}$ Alan R Berger, ${ }^{10}$ Cyril W C Kendall, ${ }^{2,11}$ John L Sievenpiper, ${ }^{1,2}$ David J A Jenkins ${ }^{1,2}$

To cite: Chiavaroli L,

Mirrahimi A, Ireland C, et al. Low-glycaemic index diet to improve glycaemic control and cardiovascular disease in type 2 diabetes: design and methods for a randomised, controlled, clinical trial. BMJ Open 2016;6: 012220. doi:10.1136/bmjopen-2016012220

- Prepublication history and additional material is available. To view please visit the journal (http://dx.doi.org/ 10.1136/bmjopen-2016012220).

Received 11 April 2016 Revised 31 May 2016 Accepted 3 June 2016

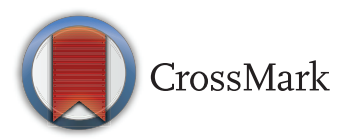

For numbered affiliations see end of article.

Correspondence to Dr David JA Jenkins; Nutritionproject@smh.ca

\section{ABSTRACT}

Introduction: Type 2 diabetes (T2DM) produces macrovascular and microvascular damage, significantly increasing the risk of cardiovascular disease (CVD), renal failure and blindness. As rates of T2DM rise, the need for effective dietary and other lifestyle changes to improve diabetes management become more urgent. Low-glycaemic index (GI) diets may improve glycaemic control in diabetes in the short term; however, there is a lack of evidence on the long-term adherence to lowGI diets, as well as on the association with surrogate markers of CVD beyond traditional risk factors. Recently, advances have been made in measures of subclinical arterial disease through the use of MRI, which, along with standard measures from carotid ultrasound (CUS) scanning, have been associated with CVD events. We therefore designed a randomised, controlled, clinical trial to assess whether low-Gl dietary advice can significantly improve surrogate markers of CVD and long-term glycaemic control in T2DM.

Methods and analysis: 169 otherwise healthy individuals with T2DM were recruited to receive intensive counselling on a low-Gl or high-cereal fibre diet for 3 years. To assess macrovascular disease, MRI and CUS are used, and to assess microvascular disease, retinal photography and 24-hour urinary collections are taken at baseline and years 1 and 3. Risk factors for CVD are assessed every 3 months. Ethics and dissemination: The study protocol and consent form have been approved by the research ethics board of St. Michael's Hospital. If the study shows a benefit, these data will support the use of low-Gl and/or high-fibre foods in the management of T2DM and its complications.

Trial Registration number: NCT01063374; Pre-results.

\section{Strengths and limitations of this study}

- This study will be the first to document the effects of a dietary intervention on measures of macrovascular disease through the detection of changes in carotid vessel wall volume assessed by MRI as a surrogate measure of cardiovascular disease, in high-risk participants.

- This study is also the longest to assess the effect of altering the dietary Gl, allowing for the exploration of sustainability of a low-Gl diet.

- The MRI images obtained from this study will provide invaluable data on the natural history of vascular disease progression in type 2 diabetes.

- A potential limitation of this study is that participants may be more health conscious than average since they are volunteering to participate in a long-term dietary trial.

- Another potential limitation is that those randomised to the control high-cereal fibre diet may have difficulty avoiding many of the healthy foods which they are told to as they are on the low-Gl diet (eg, beans and berries).

\section{INTRODUCTION}

Type 2 diabetes (T2DM) is the fastest growing chronic disease worldwide and its prevalence is projected to double over the next 20 years. Given its prevalence and heavy healthcare and quality-of-life burden, ${ }^{1}$ there is a great need for better treatment options. In uncontrolled T2DM, the macrovascular (cardiovascular disease, CVD) and microvascular (eye and kidney) risks are increased. T2DM reduces the lifespan by 10 years, ${ }^{2}$ chiefly due to CVD deaths, which are twofold 
higher in men and fourfold higher in women than in those without diabetes. ${ }^{3}$ Further, $40 \%$ of new end-stage renal disease is accounted for by diabetic nephropathy and diabetes is a major cause of renal transplants. ${ }^{4}$ Damage to retinal vessels can result in diabetic retinopathy (retinopathy), and macular oedema, which are major causes of vision loss in Western Nations. ${ }^{5}{ }^{6}$ The cost of medical care for those with diabetes in Western Nations is two to three times higher than those without diabetes, and has doubled over the past decade, ${ }^{78}$ largely related to CVD. To reduce the risk of these complications, there is a continued focus on controlling elevated blood glucose levels through lifestyle and pharmaceutical means. Prospective cohort studies and large clinical trials have demonstrated that good glycaemic control in diabetes is associated with a reduced risk of microvascular complications. $^{9-14}$ However, the results of three large randomised trials published in 2008 (ACCORD, ADVANCE and VADT) ${ }^{15-17}$ failed to show significant CVD benefit for improved glycaemic control over a 3-year to 5-year period. At the same time, concern about the cardiovascular safety of rosiglitazone and other antidiabetic medications led the US Food and Drug Administration to require demonstration of the cardiovascular safety of all new antidiabetic agents. ${ }^{18}$ Longer term follow-up of some of these trials (UKPDS and VADT), ${ }^{19} 20$ as well as the recently published EMPA REG OUTCOME study ${ }^{21}$ with the SGLT2 inhibitor, empagliflozin, have demonstrated CVD benefit. Acarbose has shown promise in reducing CVD and incident hypertension $^{22}$ when assessed as a secondary outcome in a large randomized controlled trial in participants with prediabetes, and a larger CVD study with acarbose in patients with diabetes is underway. Acarbose converts dietary carbohydrate into a slow release or low-glycaemic index (GI) food by inhibiting pancreatic amylase and small intestinal brush border sucrase-isomaltase. Low-GI foods, although recommended for diabetes control by many diabetes agencies, ${ }^{12-14}{ }^{23}$ have not been tested specifically on vascular outcomes despite cohort studies suggesting that low-GI diets, especially in women, are associated with reduced CVD. ${ }^{24}$ Additionally, a randomised trial, the PREDIMED study, that reduced the GI and GL of the $\operatorname{diet}^{25}$ and included the use of nuts and olive oil to achieve this effect also reduced CVD, especially stroke. ${ }^{26}$ We have demonstrated the greater effectiveness of low-GI diets in reducing HbAlc and blood pressure in T2DM. ${ }^{27}{ }^{28} \mathrm{We}$ therefore designed a randomised, controlled, clinical trial to assess whether dietary advice on either a low-GI or a high-cereal fibre diet will make a significant difference on carotid plaque burden and other surrogate markers of CVD, microvascular disease and long-term glycaemic control in high-risk participants with T2DM.

\section{METHODS AND ANALYSIS}

Study design

Recruitment

In 2010, potential T2DM participants were recruited through newspapers and subway advertisements, and by phoning previous study participants from our research centre who had expressed interest in further studies. After initial telephone screening, information sessions were arranged at St. Michael's Hospital. Those interested, attended a screening visit at the clinic for a blood test to determine eligibility (box 1 ). All participants gave informed consent prior to participating in any screening procedures. Physicians were contacted to ensure that those responsible for diabetes care approved participation. Those satisfying initial eligibility criteria were scheduled for a screening 2D carotid ultrasound (CUS) to assess carotid artery thickening by intima media thickness (IMT). Our original screening cut point was a maximum IMT $\geq 2.0 \mathrm{~mm}$; however, a larger than anticipated number of participants were ineligible $(\sim 66 \%$

\section{Box 1 Inclusion and exclusion criteria}

Inclusion criteria: individuals with type 2 diabetes (T2DM)

- Taking oral hypoglycaemic agents at a stable dose for $\geq 8$ weeks;

- $\mathrm{HbA} 1 \mathrm{C} \geq 6.5$ to $\leq 8.0 \%$ at initial screening visit;

- $\mathrm{HbA1C} \geq 6.5 \%$ at prestudy visit (visit just prior to randomisation);

- Diabetes diagnosed $>6$ months:

- Stable weight for 2 months (within 3\%);

- Valid Ontario Health Insurance Plan (OHIP) card and a family physician;

- If prescribed lipid medication, stable dose for $\geq 2$ weeks;

- If prescribed blood pressure medication, stable dose for $\geq 1$ week;

- Can keep written food records, with the use of a digital scale;

- Carotid maximum IMT $\geq 1.2 \mathrm{~mm}$ (originally $\geq 2.0 \mathrm{~mm}$, $\geq 1.5 \mathrm{~mm}$ in early $2012, \geq 1.2 \mathrm{~mm}$ in late 2012 ).

Exclusion criteria

- Take insulin, steroids, warfarin (Coumadin);

- Gastrointestinal disease (gastroparesis, celiac disease, ulcerative colitis, Crohn's disease, irritable bowel syndrome);

- Major cardiovascular event (stroke or myocardial infarction) or major surgery in the past 6 months;

- Major debilitating disorder;

- Clinically significant liver disease (aspartate transaminase (AST) or alanine transaminase (ALT) $>130 \mathrm{U} / \mathrm{L}$ ), excluding non-alcoholic fatty liver disease or non-alcoholic steatohepatitis;

- Hepatitis B or C;

- Renal failure (high serum creatinine $>150 \mathrm{mmol} / \mathrm{L}$ );

- Serum triglycerides $\geq 6.0 \mathrm{mmol} / \mathrm{L}$;

- History of cancer, except non-melanoma skin cancer (basal cell, squamous cell);

- Food allergies to study food components;

- Elevated blood pressure (>145/90 mm Hg) unless approved by family physician;

- Acute or chronic infections (bacterial or viral);

- Chronic inflammatory diseases (eg, rheumatoid arthritis, lupus and ulcerative colitis);

- Other conditions which in the opinion of any of the investigators would make them unsuitable for the study;

- Any condition or circumstance preventing an MRI (eg, metal workers, prostheses, metal implants or those excessively claustrophobic). 
ineligible). Therefore, in early 2012, the cut point was reduced to $\geq 1.5 \mathrm{~mm}(\sim 44 \%$ ineligible $)$ in accordance with the Mannheim carotid IMT consensus ${ }^{29}$ where $\geq 1.5 \mathrm{~mm}$ defined the beginning of atheromatous changes. It was later further reduced in late 2012 to $\geq 1.2 \mathrm{~mm}$ ( $\sim 23 \%$ ineligible), the median between the Mannheim consensus $(1.5 \mathrm{~mm})$ and the European Society of Hypertension and the European Society of Cardiology Practice Guidelines for the management of arterial hypertension $(0.9 \mathrm{~mm}){ }^{30}$ These changes allowed completion of recruitment by June 2013, while including participants with some carotid thickening. At the screening CUS scan, if the maximum IMT measure was greater than or equal to the inclusion cut point of $1.2 \mathrm{~mm}$ (or the cut point at the time of CUS scan), the sonographer also completed a 3D CUS scan.

If individuals had a recent medication change that made them ineligible at screening or prior to randomisation, they were able to return for a rescreen after the required time had elapsed.

\section{Baseline measures and randomisation}

Eligible consenting participants had a baseline carotid MRI scan at the Medical Imaging Department at Sunnybrook Health Sciences Centre. A gadolinium contrast agent, Gadovist (gadobutrol, Bayer, Mississauga, Canada), approved by Health Canada and one of several products commonly used for contrast during MRIs, was used after three safety procedures were passed. First, participants had to consent after being informed of the risks. Second, MRI safety forms were completed with the participant and reviewed by the study physician and staff at Sunnybrook. Third, forms were reviewed again by study staff at Sunnybrook immediately before the MRI. If it was deemed safe to proceed, MRI was undertaken with Gadovist. The same safety procedure was repeated for each of the two additional MRI scans at years 1 and 3 of the study. If Gadovist is deemed unsafe or the participant declines consent, the MRI scan is done without the use of Gadovist.

A baseline retinal examination was conducted by a vitreoretinal subspecialist ophthalmologist at the Department of Ophthalmology at St. Michael's Hospital and each participant had standardised seven-field diabetic retinal photographs taken.

Two baseline clinical visits occurred at St. Michael's Hospital, on average 2 weeks apart, but no more than 5. At the first baseline clinic visit, anthropometric and fasting blood measures were obtained and if HbA1c was $\geq 6.5 \%$, participants could proceed. If HbAlc was $<6.5 \%$, participants could return for a retest after 2 weeks. Participants were given detailed instruction on how to complete a 7-day food record and 24-hour urine collection using the kit provided; both were returned at the second visit.

At the second baseline clinic visit, anthropometric and fasting blood measures were again obtained and each participant was randomised to receive dietary advice on either a low-GI or high-cereal fibre diet. Randomisation was stratified by sex, HbAlc $(\leq 7.1 \%,>7.1 \%)$, smoking (yes or no) and statin use (yes or no) as documented during the first baseline visit. Participants were provided with a dietary instruction sheet based on the diet to which they were randomised (see online supplementary appendix figures S1 and S2). These diets were reviewed in detail with a study dietitian in a 30 min discussion.

Blinding the participants or the dietitians delivering the dietary advice is not possible due to the very different nature and physical form of the foods. However, it was stressed to the participants that both treatments have been considered to confer benefits in cohort studies in order to balance participant expectation of treatment benefit. To remove the possibility of bias, physicians and technical staff who are obtaining measurements are blinded to the treatments, as was the statistician who randomised the coded participants and who will analyse the data. Participants were randomised after the baseline (zero week) blood sample to avoid randomising participants who for any reason did not intend to start the study. Dropout is therefore defined as quitting post randomisation.

\section{Outcomes and study measures}

Figure 1 depicts the timing and frequency of all study measures described in detail. MRI, CUS, 24-hour urinary collections and retinal assessments are taken at baseline and years 1 and 3 with anthropometric, fasting blood measures and 7-day food records at 3-month intervals. At the 3-monthly clinic visits, the participants' medications, noting any changes, as well as any unusual or adverse events, including illness or stressful issues, that occurred since the last clinic visit are recorded in detail.

The primary outcome of the trial is change in carotid plaque volume assessed as vessel wall volume (VWV) by MRI at year 3 .

At the time of the grant submission, IMT by CUS was designated as the primary outcome based on its association with CVD when used as a screening tool. However, meta-analyse ${ }^{31-33}$ have since shown that IMT seems suboptimal for assessing changes from interventions, although it still relates overall to risk of CVD when used for screening. ${ }^{34}$ The original protocol proposed MRI of the carotid arteries as a secondary outcome. Over the last 6 years, advances in MRI technology produced high accuracy and reproducibility by minimising interoperator and intraoperator variability of image acquisition and so now make this the preferred modality for assessing change and monitoring therapeutic interventions in clinical trials. ${ }^{35-37}$ Accordingly, we adopted this as our primary outcome and adjusted our power calculations to reflect recent MRI data. ${ }^{38}$

\section{Magnetic resonance imaging}

MRI scans are being performed in the Medical Imaging Department at Sunnybrook Health Sciences Centre MRI research unit at a single site using a Philips 3-Tesla whole body scanner (Philips Healthcare, Markham, 


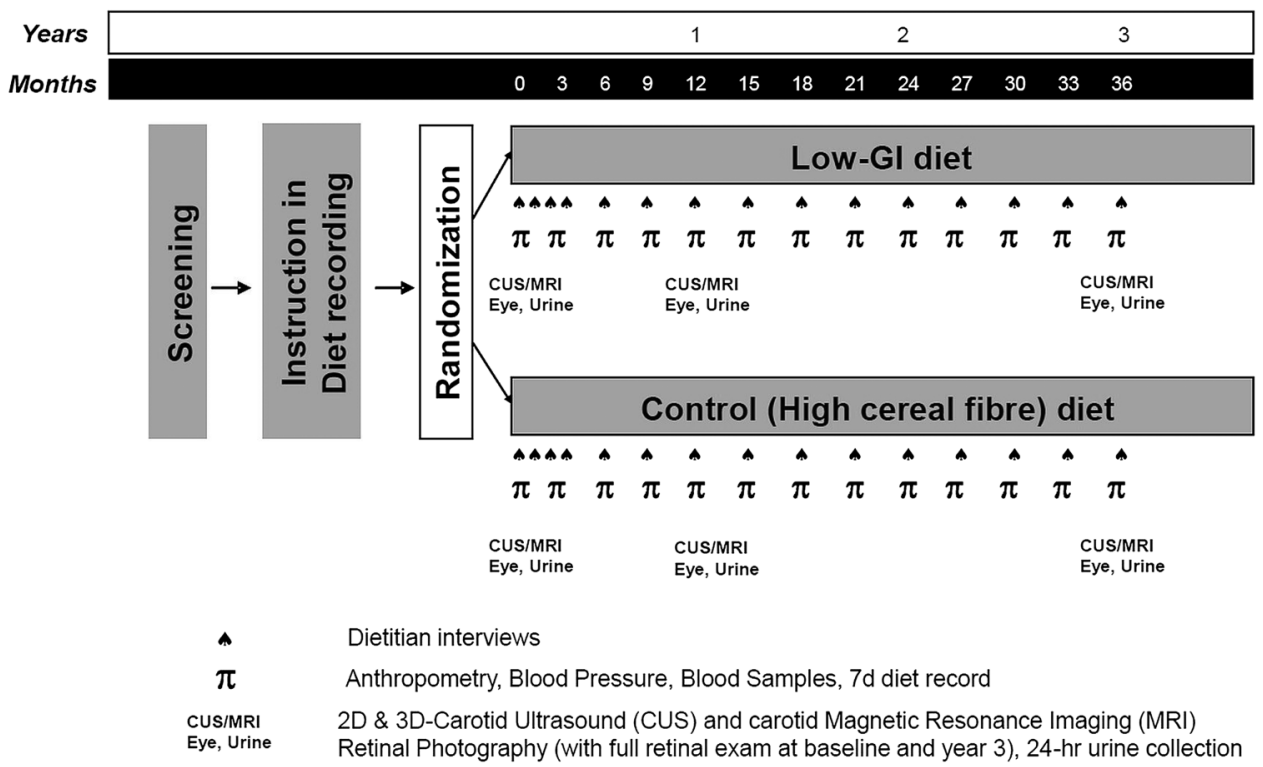

Figure 1 Schematic representation of the study protocol. Gl, glycaemic index.

Canada) with a 16-channel neurovascular coil (16-NV-SENSE). Participants are centred at the index carotid artery bifurcation as determined by carotid IMT (ie, 'index' is the side of the artery, left or right, with the highest IMT), and a shim coil covering a $10 \mathrm{~cm}$ region over the neck is used to improve magnetic field homogeneity. With a standardised protocol, six contrast weightings of the carotid artery are obtained: 2D precontrast and postcontrast enhanced T1-weighted (T1W), proton density weighted, and T2-weighted sequences, as well as 3D T1W gradient recalled echo and time of flight MR angiography sequences. Images are obtained of the left and right carotid arteries. Gadobutrol (Bayer) is used as the contrast agent when criteria are met at an intravenous injection of $0.1 \mathrm{mmol} / \mathrm{kg} \quad(0.2 \mathrm{~mL} / \mathrm{kg})$. Sequence parameters are described in online supplementary appendix table $\mathrm{S} 1$. Total scan time is an average of $60 \mathrm{~min}$ and allows coverage of 2D imaging capturing a $32 \mathrm{~mm}$ segment $(2 \mathrm{~mm}$ thickness $\times 16$ matched images among the four weightings), while 3D imaging captures the entire carotid artery from its origin to the Circle of Willis. The cardiovascular imaging software, VesselMASS (Medis, the Netherlands), is used for image analysis. Image grading is performed during the analysis (see online supplementary appendix table S2) and images with poor quality (grade $<3$ ) or with missing images will be excluded from analyses. Location matching of the available MR images is performed using the baseline index carotid artery over the different time points before lumen and outer wall contours are identified. VWV is automatically generated from the software. $^{39-41}$ Intraclass correlation values for measurements are above 0.9 (good to excellent) $;^{42-44}$ however, a single, trained and blinded reader will assess all measurements.

MRI will also allow assessment of several secondary outcomes that are surrogate markers of CVD: changes in intraplaque haemorrhage, lipid-rich necrotic core and carotid artery calcium status, ${ }^{37} 384546$ each of which has an intraclass correlation value for measurements above 0.9 (good to excellent). ${ }^{42} 44$

\section{B-mode carotid ultrasound (CUS)}

An additional secondary outcome assessing macrovascular disease is IMT by 2D B-mode CUS, a measure that is related to CVD risk. ${ }^{34}$

Standardised CUS scanning and reading protocols are used following a similar protocol to the ACAPS ${ }^{4-49}$ and SECURE $^{50} 51$ trials, which was validated in ACAPS. ${ }^{49}$ CUS imaging and reading is performed by two trained and certified sonographers who are unaware of the treatment assignment on a Philips iU22 Ultrasound system (Philips Healthcare, Andover, Massachusetts, USA) at Sunnybrook. Most participants are scanned with the L9-3 linear high-frequency transducer; however, when the vessel is very superficial, the $12-5$ transducer is used in order to visualise the near wall. Each participant is positioned supine on the examination table, with the neck extended and rotated away from the side of interest. Transverse and longitudinal views of each carotid artery are obtained from the common carotid artery to the bifurcation and the internal carotid artery (ICA). The ICA is differentiated from the external carotid artery based on its low resistance spectral Doppler flow pattern. On the longitudinal view, each carotid artery is divided into three segments $1 \mathrm{~cm}$ long defined relative to the carotid flow divider (figure 2). Twelve IMT measurements of the near and far walls at each carotid segment are made using electronic calipers and the mean maximum IMT is computed as the average of the segment maximum IMTs.

Further secondary outcomes significantly related to CVD risk include anthropometric, urine and blood measures, ${ }^{52}$ which will be analysed as previously described. ${ }^{28}$ Briefly, anthropometric data include body weight, seated blood pressure measured as the mean of triplicate 


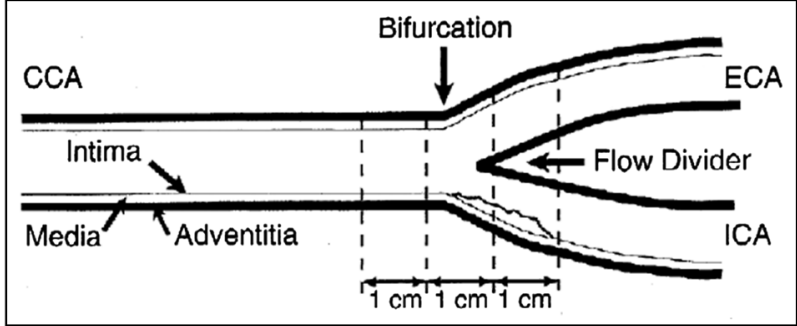

Figure 2 Diagrammatic example of a right common carotid artery scan. The far wall of the bifurcation segment as well as part of the internal carotid artery are narrowed by a plaque. Adapted from Lonn et al. ${ }^{51}$

measures made with an automatic sphygmomanometer (Omron HEM 907XL, OMRON Healthcare, Burlington, Ontario, Canada), and waist (at the umbilicus, 2 inches above and lying down) and hip circumference. Twenty-four-hour urine is collected by discarding the first urine on waking, noting the time and then collecting each subsequent excretion up to and including the first void at the same time on waking the next morning. Urinary measures include C-peptide, urea, creatinine and electrolytes. Blood measures include $\mathrm{HbAlc}$, fasting glucose, fasting lipids and liver function.

Other study outcomes include $50^{\circ}$ stereoscopic colour fundus photographs of seven standard fields (retinal photography) to assess degree of retinopathy following a similar protocol to the ACCORD study. ${ }^{53}$ Vitreoretinal subspecialist ophthalmologists blinded to the intervention will read the photos to assess any changes.

Dietary assessments are being made using participant completed 7-day food records that are analysed using a computer program (ESHA Food Processor SQL V.10.9; ESHA, Salem, Oregon, USA) based on a United States Department of Agriculture (USDA) database, ${ }^{54}$ supplemented with the Canada Nutrient File, ${ }^{55}$ with GI values from international GI tables, ${ }^{56}$ substituted with GI testing through the University of Toronto at Glycemic Index Laboratories, Canada, using the bread scale (where bread $=100$; for the glucose scale, bread scale values are multiplied by 0.71). Product data are updated with manufacturers' nutrient information and with relevant foods analysed by Covance Laboratories (Madison, Wisconsin, USA).

\section{Interventions}

Participants were randomised to receive dietary advice on either a low-GI or high-cereal fibre diet for 3 years together with advice on the National Cholesterol Education Program Adult Treatment Panel III (NCEP ATP III) diet. Diet histories recorded for the 7 days prior to clinic visits are being assessed for detail and consistency by the dietitian in the participant's presence, and used to guide dietary advice. Assessment is made of satiety (using a 9-point bipolar semantic scale) and on palatability and sustainability (using 10-point scales).

Both diets conform as closely as possible to NCEP ATP III guidelines with $<7 \%$ saturated fat and $<200 \mathrm{mg}$ dietary cholesterol daily ${ }^{57}$ and provide the same level of fibre (up to $35 \mathrm{~g}$ /day). Advice encouraging all participants to reach ideal body weight is standard advice for those with diabetes, $85-90 \%$ of whom are overweight, together with encouragement to exercise at a level they can sustain prior to and over the course of the study. The consistency of exercise is checked at each clinic visit and any deviations recorded.

Low-GI dietary advice encourages use of intact grains, including specific low-GI breads, pasta, parboiled rice, coarse cut oats, Red River and All Bran Buds with psyllium breakfast cereals, cooked dried or canned peas, beans or lentils, barley and low-GI temperate climate fruit, including apples, oranges and berries (see online supplementary appendix figure S1).

High-cereal fibre dietary advice encourages use of whole grains, including whole wheat breads, wheat fibre cereals, cream of wheat hot cereal, brown rice and tropical fruit, including bananas, mangos and pineapples (see online supplementary appendix figure S2). Sample nutritional profiles of each diet demonstrating a 13-unit difference in GI between test and control as demonstrated in our previous 6-month trial ${ }^{27}$ is provided in online supplementary appendix table S3.

Dietary advice is provided through half-hour individual sessions with the dietitian every 3 months at clinic visits, as well as through monthly phone calls for the first 3 months and thereafter, at least one 10 minute phone interview for 1-day diet recalls between dietitian interviews, with additional phone interviews for those with poor $(<75 \%)$ adherence to the study protocol (ie, poor diet and missed visits).

\section{Sample size}

Our original power calculation was based on IMT by CUS which was the original primary end point. We had estimated that 160 participants would need to be randomised with 120 participants completing the study (25\% attrition). A total of 169 participants were randomised.

For our primary outcome, VWV by MRI, the magnitude of difference between the groups that can be seen with 169 randomised participants was calculated using the estimate of variance of the measurement as observed $(\mathrm{SD}=252)$ in Saam et al. ${ }^{38}$ This showed that a treatment difference of $10 \%$ can be detected with $80 \%$ power and $\alpha=0.05$, assuming a $25 \%$ attrition.

Furthermore, with a sample size of 160 participants, we will be able to detect changes in the important secondary end point, HbA1c, which could influence arterial damage. For HbAlc, if 120 of the randomised 169 participants complete the study, we will be able to detect a $0.3 \%$ treatment difference with a pooled SD of the treatment difference of 0.578 at a two-tailed significance level $(\alpha)=0.05$ and power $(1-\beta)=0.8$ using a twotreatment parallel design, with an independent-samples t-test. This calculation is based on our previous study in T2DM using similar treatments over a 6-month period. $^{27}$ 


\section{Statistical analysis planned}

All randomised participants will be included in the intention-to-treat analyses. Results will be expressed as means \pm SEM or $95 \%$ CIs.

\section{Primary analysis}

The primary analysis will assess the between-treatment difference in change from baseline in VWV, where change is assessed as years 1 and 3 adjusted for baseline using a repeated-measures mixed model (PROC MIXED in SAS 9.4) (SAS Institute: SAS/STAT Proprietary Software 9.4. Cary, NC: SAS Institute; 2002-2012) in an intention-to-treat analysis, without adjustment for covariates. Every effort will be made to obtain final vascular imaging and blood samples from those who do not provide these in clinic due to attrition or loss to follow-up.

\section{Sensitivity analysis}

Robustness will be assessed of our primary finding to model assumptions: (1) To address the impact of potential imbalance in prognostic factors, we will repeat the primary analysis using mixed models but controlling for age, sex, duration of diabetes, waist circumference, cholesterol medication use, baseline VWV, smoking, blood pressure and family history of CVD, together with dietary variables (baseline GI, saturated fat, dietary cholesterol, dietary pulse and nut intake). Missing data for covariates will be handled using the missing indicator method; (2) To assess the robustness of our primary analysis of missing data, we will repeat the primary analysis using completer and per-protocol analyses, and multiple imputations to generate missing data and (3) To assess the impact of participant-level factors on the primary outcome, we will examine changes in indices of vascular damage separately in (a) those who meet HbAlc target versus those with less good glycaemic control (eg, $\mathrm{HbAlc} \leq 7 \%$ vs $\mathrm{HbAlc}>7 \%$ at the end of the study); (b) those with good compliance, that is, by quartiles of change in GI at years 1 and 3, those in the highest quartiles of specific low-GI components (dietary pulses, temperate climate fruit, nuts, etc) and high-fibre completers by quartiles of change in fibre and (c) by IMT at study entry. Exploratory assessment of the significance of between-subgroup changes in the primary outcome will be undertaken with Wald tests of the interaction terms.

\section{Exploratory analyses}

We anticipate that this study will yield a rich data set. We have therefore mapped out some exploratory analyses: (1) to assess response trajectories, by comparing treatment slopes across all post-treatment measures, that is, an assessment of whether year 1 values differ from year 3 values; (2) to assess treatment differences in medication use over all post-treatment values; (3) further analyses will examine causal pathways between diet, metabolic parameters and measures of arterial function using methods of path analysis and structural equation modelling, as appropriate.

\section{ETHICS AND DISSEMINATION}

The protocol and consent forms were approved by the research ethics board of St. Michael's Hospital. The study was registered with clinicaltrials.gov (identifier: NCT01063374).

\section{Participant safety}

Privacy will be enhanced by data deidentification. Databases with personal health information will be password protected. Paper forms with personal health information (eg, participant charts) will be kept in locked cabinets and in locked rooms, and the department door locked after hours.

Electronic files will identify participants only through identification codes. Access to paper and electronic data files is limited to the principle investigator (PI), statisticians, dietitians, students and data entry personnel working on the project.

A separate chart with routine clinical information is maintained for contact with participants' family physicians and will be accessible only to the PI and study staff.

A Data Safety Monitoring Board will periodically review (approximately once per year or when safety issues arise) the progress of the trial to oversee participant safety and provide advice on the status and continuation of the overall study.

HbA1c is reviewed at each visit and as necessary with one of the physicians of the Safety Committee who are not involved in the day-to-day running of the trial (RGJ, LAL) with participants identified only by code. Participants will be referred to his/her family physician for treatment if HbA1c exceeds the $8.5 \%$ threshold of recommended targets for glycaemic control, according to diabetes guidelines, ${ }^{11}{ }^{12}$ on two successive occasions, or if hypoglycaemic symptoms associated with low blood glucose levels occur. If a participant's physician considers that a change in dosage or medication is required, a predetermined paradigm will be used, and the Safety Committee notified. Blood pressure is also reviewed. If levels surpassed the screening cut-off of 145/90 mm Hg, the participant's physician is notified. Adverse events will be dealt with on a case-by-case basis depending on whether the situation is likely to resolve spontaneously or whether medical intervention is required.

\section{Discussion and implications}

This study will be the first to document the effects of a dietary intervention on measures of macrovascular disease through the detection of changes in carotid VWV as a surrogate measure of CVD, in high-risk participants. We believe this will open the way for other investigators to use vascular MRI in their research and for use in clinical practice as a diagnostic tool and especially for follow-up. It is also the longest trial to date to assess the 
effect of altering the dietary GI and will also provide invaluable data on the natural history of vascular disease. In view of the global rise in the incidence of T2DM and the associated vascular complications, including increased CVD risk, therapeutic approaches that address microvascular and macrovascular risk factors are now urgently required.

At present, diabetes is an immense burden on the healthcare system of Western Nations, projected to continue to increase, and largely related to CVD and microvascular complications. ${ }^{78}$ A $25 \%$ sparing of T2DM microvascular complications would result long term from a $0.9 \%$ reduction in HbAlc according to the UKPDS data. ${ }^{58}$ We achieved a $0.5 \%$ reduction in HbAlc in our previous 6-month randomised, controlled trial of similar low-GI dietary advice in those with T2DM, ${ }^{27}$ so we expect a similar reduction in this investigation. The economic (let alone social) impact of even one-third the reduction in HbA1c seen in the $\mathrm{UKPDS}^{58}$ is likely to be considerable. If low-GI diets can be shown to reduce macrovascular disease risk factors in addition to reducing microvascular disease, results will not only increase understanding of the role of diet and nutrition in macrovascular disease development in T2DM but will also influence guidelines for the treatment of diabetes and the nature of the products produced by the food industry, and the savings in cost and suffering will be significant.

\author{
Author affiliations \\ ${ }^{1}$ Department of Nutritional Sciences, Faculty of Medicine, University of \\ Toronto, Toronto, Ontario, Canada \\ ${ }^{2}$ Clinical Nutrition and Risk Factor Modification Center, St. Michael's Hospital, \\ Toronto, Ontario, Canada \\ ${ }^{3}$ Faculty of Health Sciences, School of Medicine, Queen's University, Kingston, \\ Ontario, Canada \\ ${ }^{4}$ Department of Medical Imaging, Sunnybrook Health Sciences Centre, \\ Toronto, Ontario, Canada \\ ${ }^{5}$ Institute of Medical Science, University of Toronto, Toronto, Ontario, Canada \\ ${ }^{6}$ Department of Clinical Epidemiology and Biostatistics, Faculty of Health \\ Sciences, McMaster University, Hamilton, Ontario, Canada \\ ${ }^{7}$ National Cancer Institute "Fondazione G. Pascale", Naples, Italy \\ ${ }^{8}$ Division of Endocrinology and Metabolism, St. Michael's Hospital, Toronto, \\ Ontario, Canada \\ ${ }^{9}$ Dalla Lana School of Public Health, University of Toronto, Toronto, Ontario, \\ Canada \\ ${ }^{10}$ Department of Ophthalmology, St. Michael's Hospital, Toronto, Ontario, \\ Canada \\ ${ }^{11}$ College of Pharmacy and Nutrition, University of Saskatchewan, Saskatoon, \\ Saskatchewan, Canada
}

Acknowledgements The authors thank Dr Rajeev Muni for his help as one of our vitreoretinal subspecialist ophthalmologists from the Department of Ophthalmology at St. Michael's Hospital. MRI contrast was provided by Bayer Healthcare (Bayer, Mississauga, Canada).

Contributors DJAJ, LAL, RGJ, GMK-E, ARM, CWCK and JLS conceived the study and wrote the study protocol. LC, AM, CI, SM, SS-P, JC, 00, TM, DP, RJdS, LSAA, BB, SBM, SKN, ARM, ARB and DJAJ contributed significantly to the acquisition of the data. LC, AM, CI, RGJ, GMK-E, ARM and DJAJ significantly contributed to the planning of analyses of the data. LC and DJAJ drafted the manuscript and all authors revised the manuscript critically for important intellectual content. All authors reviewed and approved the final manuscript and agree to be accountable for all aspects of the work.
Funding This work was supported by the Canadian Institutes of Health Research (CIHR), as well as Loblaws Inc., and Barilla. DJAJ was funded by the Government of Canada through the Canada Research Chair Endowment. None of the funders had a role in the study design, collection, analysis or interpretation of the data; in the writing of the report; and in the decision to submit the paper for publication.

Competing interests LC has received research support from the Canadian Institutes of Health Research (CIHR). LC is a clinical research coordinator and SKN is a clinical research dietitian at Glycemic Index Laboratories, Toronto, Ontario, Canada. RJdS is funded by a CIHR Postdoctoral Fellowship Award and has received research support from the CIHR, the Calorie Control Council, the Canadian Foundation for Dietetic Research and The Coca-Cola Company (investigator initiated, unrestricted grant). RJdS has served as an external resource person to WHO's Nutrition Guidelines Advisory Group and received travel support from the WHO to attend group meetings. RJdS is the lead author of systematic reviews and meta-analyses commissioned by the WHO on the relation of SFAs and trans-fatty acids with health outcomes. LSAA has received an honorarium from the Nutrition Foundation of Italy (NFI) to co-organise a glycaemic index summit. ARM is currently consultant for Jansen/Johnson and Johnson. JLS has received research support from the CIHR, American Society of Nutrition (ASN), Canadian Diabetes Association (CDA), Banting \& Best Diabetes Centre (BBDC), Calorie Control Council, The Coca-Cola Company (investigator initiated, unrestricted), Dr Pepper Snapple Group (investigator initiated, unrestricted), Pulse Canada and the International Tree Nut Council Nutrition Research and Education Foundation. He has received travel funding, speaker fees and/or honoraria from American Heart Association (AHA), American College of Physicians (ACP), ASN, National Institute of Diabetes and Digestive and Kidney Diseases (NIDDK) of the National Institutes of Health (NIH), CDA, CNS, University of South Carolina, University of Alabama at Birmingham, Oldways Preservation Trust, NFI, Calorie Control Council, Diabetes and Nutrition Study Group of the European Association for the Study of Diabetes (EASD), International Life Sciences Institute (ILSI) North America, ILSI Brazil, Abbott Laboratories, Pulse Canada, Canadian Sugar Institute, Dr Pepper Snapple Group, The Coca-Cola Company, Corn Refiners Association, World Sugar Research Organization, Dairy Farmers of Canada and Società Italiana di Nutrizione Umana (SINU). He has consulting arrangements with Winston \& Strawn LLP, Perkins Coie LLP and Tate \& Lyle. $\mathrm{He}$ is on the Clinical Practice Guidelines Expert Committee for Nutrition Therapy of the CDA and EASD, as well as being on an ASN writing panel for a scientific statement on sugars. He is a member of the International Carbohydrate Quality Consortium (ICQC) and Board Member of the Diabetes and Nutrition Study Group of the EASD. He serves an unpaid scientific advisor for the International Life Science Institute (ILSI) North America, Food, Nutrition, and Safety Program (FNSP) and the Committee on Carbohydrates. His wife is an employee of Unilever Canada. CWCK has received research grants, travel funding, consultant fees, honoraria and served on the scientific advisory board for Abbott Laboratories, Advanced Food Materials Network, Agriculture and Agri-Food Canada (AAFC), Almond Board of California, American Peanut Council, American Pistachio Growers, Barilla, Bayer, California Strawberry Commission, Calorie Control Council, CIHR, Canola Council of Canada, The Coca-Cola Company, Danone, General Mills, Hain Celestial, International Nut and Dried Fruit Council, International Tree Nut Council Nutrition Research and Education Foundation, Kellogg, Kraft, Loblaw Brands Ltd, NFI, Oldways Preservation Trust, Orafti, Paramount Farms, Peanut Institute, Pepsi-Co, Pulse Canada, Sabra Dipping Co., Saskatchewan Pulse Growers, Solae, Sun-Maid, Tate \& Lyle, Unilever and White Wave Foods. He is on the Dietary Guidelines Committee for the Diabetes Nutrition Study Group of the European Association for the Study of Diabetes. DJAJ has received research grants from Saskatchewan Pulse Growers, the Agricultural Bioproducts Innovation Program through the Pulse Research Network, the Advanced Foods and Material Network, Loblaw Companies Ltd., Unilever, Barilla, the Almond Board of California, Agriculture and Agri-food Canada, Pulse Canada, Kellogg's Company, Canada, Quaker Oats, Canada, Procter \& Gamble Technical Centre Ltd., Bayer Consumer Care, Springfield, New Jersey, Pepsi/Quaker, International Nut \& Dried Fruit (INC), Soy Foods Association of North America, the Coca-Cola Company (investigator initiated, unrestricted grant), Solae, Haine Celestial, the Sanitarium Company, Orafti, the International Tree Nut Council Nutrition Research and Education Foundation, the Peanut Institute, the Canola and Flax Councils of Canada, the CCC, the 
CIHR, the Canada Foundation for Innovation and the Ontario Research Fund. He has been on the speaker's panel, served on the scientific advisory board and/or received travel support and/or honoraria from the Almond Board of California, Canadian Agriculture Policy Institute, Loblaw Companies Ltd, the Griffin Hospital (for the development of the NuVal scoring system, the Coca-Cola Company, EPICURE, Danone, Saskatchewan Pulse Growers, Sanitarium Company, Orafti, the Almond Board of California, the American Peanut Council, the International Tree Nut Council Nutrition Research and Education Foundation, the Peanut Institute, Herbalife International, Pacific Health Laboratories, Nutritional Fundamental for Health, Barilla, Metagenics, Bayer Consumer Care, Unilever Canada and Netherlands, Solae, Kellogg, Quaker Oats, Procter \& Gamble, the Coca-Cola Company, the Griffin Hospital, Abbott Laboratories, the Canola Council of Canada, Dean Foods, the California Strawberry Commission, Haine Celestial, PepsiCo, the Alpro Foundation, Pioneer Hi-Bred International, DuPont Nutrition and Health, Spherix Consulting and WhiteWave Foods, the Advanced Foods and Material Network the Canola and Flax Councils of Canada, the Nutritional Fundamentals for Health, Agri-Culture and Agri-Food Canada, the Canadian Agri-Food Policy Institute, Pulse Canada, the Saskatchewan Pulse Growers, the Soy Foods Association of North America, the NFI, Nutra-Source Diagnostics, the McDougall Program, the Toronto Knowledge Translation Group (St. Michael's Hospital), the Canadian College of Naturopathic Medicine, The Hospital for Sick Children, the Canadian Nutrition Society (CNS), the ASN, Arizona State University, Paolo Sorbini Foundation and the Institute of Nutrition, Metabolism and Diabetes. He received an honorarium from the US Department of Agriculture to present the 2013 W.0. Atwater Memorial Lecture. He received the 2013 Award for Excellence in Research from the International Nut and Dried Fruit Council. He received funding and travel support from the Canadian Society of Endocrinology and Metabolism to produce mini cases for the CDA. $\mathrm{He}$ is a member of the ICQC. His wife, Alexandra $L$ Jenkins, is a director and partner of Glycemic Index Laboratories, Inc., and his sister received funding through a grant from the St. Michael's Hospital Foundation to develop a cookbook for one of his studies.

Open Access This is an Open Access article distributed in accordance with the Creative Commons Attribution Non Commercial (CC BY-NC 4.0) license, which permits others to distribute, remix, adapt, build upon this work noncommercially, and license their derivative works on different terms, provided the original work is properly cited and the use is non-commercial. See: http:// creativecommons.org/licenses/by-nc/4.0/

\section{REFERENCES}

1. International Diabetes Federation (IDF). Diabetes atlas. 6th edn 2013. http://www.idf.org/diabetesatlas (accessed $10 \mathrm{Jul}$ 2015).

2. Manuel DG, Schultz SE. Health-related quality of life and health-adjusted life expectancy of people with diabetes in Ontario, Canada, 1996-1997. Diabetes Care 2004;27:407-14.

3. Public Health Agency of Canada. Unpublished analysis using 2008/ 09 data from the Canadian Chronic Disease Surveillance System (Public Health Agency of Canada). 2011. http://www.phac-aspc.gc. ca/cd-mc/cvd-mcv/index-eng.php

4. Motilch ME, De Fronzo RA, Franz MJ, American Diabetes Association. Diabetic nephropathy. Diabetes Care 2003;26(Suppl 1): S94-8.

5. CNIB. The Cost of Vision Loss in Canada. CNIB and the Canadian Ophthalmological Society. Access Economics. 2009. http://www. cnib.ca/eng/cnib\%20document\%20library/research/covl_full_report. pdf (accessed Jul 10 2015).

6. Centers for Disease Control and Prevention (CDC). Vision Health Initiative. National Center for Health Statistics, Division of Health Interview Statistics, data from the National Health Interview Survey. 2015. http://www.cdc.gov/visionhealth/basic_information/eye_ disorders.htm (accessed 10 Jul 2015).

7. Canadian Diabetes Association. An economic tsunami: the cost of diabetes in Canada. 2009:1-21. http://www.diabetes.ca/publicationsnewsletters/advocacy-reports/economic-tsunami-the-cost-ofdiabetes-in-canada (accessed 10 Jul 2015).

8. American Diabetes Association. Economic costs of diabetes in the U.S. in 2012. Diabetes Care 2013;36:1033-46.

9. The Diabetes Control and Complications Trial Research Group. The relationship of glycemic exposure $(\mathrm{HbA1c})$ to the risk of development and progression of retinopathy in the diabetes control and complications trial. Diabetes 1995;44:968-83.

10. Stratton IM, Adler Al, Neil HA, et al. Association of glycaemia with macrovascular and microvascular complications of type 2 diabetes (UKPDS 35): prospective observational study. BMJ 2000;321:405-12.

11. Imran SA, Rabasa-Lhoret R, Ross S Canadian Diabetes Association Clinical Practice Guidelines Expert Committee. Targets for glycemic control. Can J Diabetes 2013;37(Suppl 1):S31-4.

12. American Diabetes Association. 6. Glycemic targets. Diabetes Care 2015;38(Suppl 1):S33-40.

13. International Diabetes Federation 2012 Clinical Guidelines Task Force. Global guidelines for Type 2 diabetes. 2012 http://www.idf.org

14. Colagiuri S, Dickinson S, Girgis S, et al. National evidence based guideline for blood glucose control in type 2 diabetes. Canberra: Diabetes Australia and the NHMRC, 2009.

15. Gerstein HC, Miller ME, Byington RP, et al. Action to Control Cardiovascular Risk in Diabetes Study Group. Effects of intensive glucose lowering in type 2 diabetes. $N$ Engl J Med 2008;358:2545-59.

16. Duckworth W, Abraira C, Moritz T, et al. Glucose control and vascular complications in veterans with type 2 diabetes. $N$ Engl $J$ Med 2009;360:129-39.

17. Patel A, MacMahon S, Chalmers J, et al., Advance Collaborative Group. Intensive blood glucose control and vascular outcomes in patients with type 2 diabetes. N Engl J Med 2008;358:2560-72.

18. US Food and Drug Administration. Guidance for industry: diabetes mellitus: developing drugs and therapeutic biologics for treatment and prevention. 2008. http://www.fda.gov/cder/guidance/7630dft.pdf (released 13 Feb 2008).

19. Hayward RA, Reaven PD, Wiitala WL, et al. Follow-up of glycemic control and cardiovascular outcomes in type 2 diabetes. $N$ Engl J Med 2015;372:2197-206.

20. Holman RR, Paul SK, Bethel MA, et al. 10-Year follow-up of intensive glucose control in type 2 diabetes. $N$ Engl J Med 2008;359:1577-89.

21. Zinman B, Wanner C, Lachin JM, et al. Empagliflozin, cardiovascula outcomes, and mortality in type 2 diabetes. $N$ Engl J Med 2015;373:2117-28

22. Chiasson JL, Josse RG, Gomis R, et al. Acarbose treatment and the risk of cardiovascular disease and hypertension in patients with impaired glucose tolerance: the STOP-NIDDM trial. JAMA 2003;290:486-94

23. Dworatzek PD, Arcudi K, Gougeon R, et al. Canadian Diabetes Association Clinical Practice Guidelines Expert Committee. Nutrition therapy. Can J Diabetes 2013;37(Suppl 1):S45-55.

24. Mirrahimi A, de Souza RJ, Chiavaroli L, et al. Associations of glycemic index and load with coronary heart disease events: a systematic review and meta-analysis of prospective cohorts. J Am Heart Assoc 2012;1:e000752.

25. Rodriguez-Rejon Al, Castro-Quezada I, Ruano-Rodriguez C, et al. Effect of a Mediterranean diet intervention on dietary glycemic load and dietary glycemic index: the PREDIMED study. $J$ Nutr Metab 2014;2014:985373.

26. Estruch R, Ros E, Salas-Salvado J, et al. Primary prevention of cardiovascular disease with a Mediterranean diet. $N$ Engl J Med 2013;368:1279-90.

27. Jenkins DJ, Kendall CW, McKeown-Eyssen G, et al. Effect of a low-glycemic index or a high-cereal fiber diet on type 2 diabetes: a randomized trial. JAMA 2008;300:2742-53.

28. Jenkins DJ, Kendall CW, Augustin LS, et al. Effect of legumes as part of a low glycemic index diet on glycemic control and cardiovascular risk factors in type 2 diabetes mellitus: a randomized controlled trial. Arch Intern Med 2012;172:1653-60.

29. Touboul PJ, Hennerici MG, Meairs S, et al. Mannheim carotid intima-media thickness consensus (2004-2006). An update on behalf of the Advisory Board of the 3rd and 4th Watching the Risk Symposium, 13th and 15th European Stroke Conferences, Mannheim, Germany, 2004, and Brussels, Belgium, 2006. Cerebrovascular Dis 2007;23:75-80.

30. Mancia G, De Backer G, Dominiczak A, et al. 2007 Guidelines for the management of arterial hypertension: the task force for the management of arterial hypertension of the European Society of Hypertension (ESH) and of the European Society of Cardiology (ESC). J Hypertens 2007;25:1105-87.

31. Lorenz MW, Price JF, Robertson C et al. Carotid intima-media thickness progression and risk of vascular events in people with diabetes: results from the PROG-IMT collaboration. Diabetes Care 2015;38:1921-9. 
32. Lorenz MW, Polak JF, Kavousi M, et al. Carotid intima-media thickness progression to predict cardiovascular events in the general population (the PROG-IMT collaborative project): a meta-analysis of individual participant data. Lancet 2012;379:2053-62.

33. Costanzo P, Perrone-Filardi P, Vassallo E, et al. Does carotid intima-media thickness regression predict reduction of cardiovascular events? A meta-analysis of 41 randomized trials. J Am Coll Cardiol 2010;56:2006-20.

34. Polak JF, Pencina MJ, Meisner A, et al. Associations of carotid artery intima-media thickness (IMT) with risk factors and prevalent cardiovascular disease: comparison of mean common carotid artery IMT with maximum internal carotid artery IMT. J Ultrasound Med 2010;29:1759-68.

35. Underhill HR, Yuan C, Yarnykh VL, et al. Arterial remodeling in [corrected] subclinical carotid artery disease. JACC CardiovasC Imaging 2009;2:1381-9.

36. Underhill HR, Yuan C. Carotid MRI: a tool for monitoring individual response to cardiovascular therapy? Expert Rev Cardiovasc Ther 2011;9:63-80.

37. Singh N, Moody AR, Roifman I, et al. Advanced MRI for carotid plaque imaging. Int $J$ Cardiovasc Imaging 2016;32:83-9.

38. Saam T, Kerwin WS, Chu B, et al. Sample size calculation for clinical trials using magnetic resonance imaging for the quantitative assessment of carotid atherosclerosis. J Cardiovasc Magn Reson 2005;7:799-808.

39. van't Klooster R, de Koning PJ, Dehnavi RA, et al. Automatic lumen and outer wall segmentation of the carotid artery using deformable three-dimensional models in MR angiography and vessel wall images. J Magn Reson Imaging 2012;35:156-65.

40. van't Klooster R, Staring M, Klein S, et al. Automated registration of multispectral MR vessel wall images of the carotid artery. Med Phys 2013:40:121904.

41. van't Klooster R, Truijman MT, van Dijk AC, et al. Visualization of local changes in vessel wall morphology and plaque progression in serial carotid artery magnetic resonance imaging. Stroke 2014;45: e160-3.

42. Li F, Yarnykh VL, Hatsukami TS, et al. Scan-rescan reproducibility of carotid atherosclerotic plaque morphology and tissue composition measurements using multicontrast MRI at 3T. J Magn Reson Imaging 2010;31:168-76.

43. Duivenvoorden $\mathrm{R}$, de Groot $\mathrm{E}$, Elsen $\mathrm{BM}$, et al. In vivo quantification of carotid artery wall dimensions: 3.0-Tesla MRI versus B-mode ultrasound imaging. Circ Cardiovasc Imaging 2009;2:235-42.

44. Sun J, Zhao XQ, Balu N, et al. Carotid magnetic resonance imaging for monitoring atherosclerotic plaque progression: a multicenter reproducibility study. Int J Cardiovasc Imaging 2015;31:95-103.

45. Tardif JC, Spence JD, Heinonen TM, et al. Atherosclerosis imaging and the Canadian Atherosclerosis Imaging Network. Can J Cardiol 2013;29:297-303.
46. Moody AR, Singh N. Incorporating carotid plaque imaging into routine clinical carotid magnetic resonance angiography. Neuroimaging Clin N Am 2016;26:29-44.

47. Furberg CD, Adams HP Jr, Applegate WB, et al. Effect of lovastatin on early carotid atherosclerosis and cardiovascular events. Asymptomatic Carotid Artery Progression Study (ACAPS) Research Group. Circulation 1994;90:1679-87.

48. Pitt $B$, Byington RP, Furberg CD, et al. Effect of amlodipine on the progression of atherosclerosis and the occurrence of clinical events. PREVENT Investigators. Circulation 2000;102: 1503-10.

49. Riley WA, Barnes RW, Applegate WB, et al. Reproducibility of noninvasive ultrasonic measurement of carotid atherosclerosis. The Asymptomatic Carotid Artery Plaque Study. Stroke 1992;23:1062-8.

50. Lonn E, Yusuf S, Dzavik V, et al. Effects of ramipril and vitamin E on atherosclerosis: the study to evaluate carotid ultrasound changes in patients treated with ramipril and vitamin E (SECURE). Circulation 2001;103:919-25.

51. Lonn EM, Yusuf $\mathrm{S}$, Doris $\mathrm{Cl}$, et al. Study design and baseline characteristics of the study to evaluate carotid ultrasound changes in patients treated with ramipril and vitamin E: SECURE. Am J Cardiol 1996;78:914-19.

52. Anderson TJ, Grégoire J, Hegele RA, et al. 2012 update of the Canadian Cardiovascular Society guidelines for the diagnosis and treatment of dyslipidemia for the prevention of cardiovascular disease in the adult. Can J Cardiol 2013;29: 151-67.

53. Ismail-Beigi $\mathrm{F}$, Craven $\mathrm{T}$, Banerii MA, et al. Effect of intensive treatment of hyperglycaemia on microvascular outcomes in type 2 diabetes: an analysis of the ACCORD randomised trial. Lancet 2010;376:419-30.

54. U.S. Department of Agriculture. Composition of Foods, Agriculture Handbook No. 23: The Agriculture Research Service. Washington, DC, U.S. Dept of Agriculture, 2010.

55. Canadian Nutrient File. http://webprod3.hc-sc.gc.ca/cnf-fce/ index-eng.jsp

56. Atkinson FS, Foster-Powell K, Brand-Miller JC. International tables of glycemic index and glycemic load values: 2008. Diabetes Care 2008;31:2281-3.

57. Summary of the second report of the National Cholesterol Education Program (NCEP) Expert Panel on Detection, Evaluation, and Treatment of High Blood Cholesterol in Adults (Adult Treatment Panel II). JAMA 1993;269:3015-23.

58. Intensive blood-glucose control with sulphonylureas or insulin compared with conventional treatment and risk of complications in patients with type 2 diabetes (UKPDS 33). UK Prospective Diabetes Study (UKPDS) Group. Lancet 1998;352: 837-53. 